\title{
Numerical detection of unstable periodic orbits in continuous-time dynamical systems with chaotic behaviors
}

\author{
Y. Saiki \\ Research Institute for Mathematical Sciences, Kyoto University, Japan
}

Received: 2 July 2007 - Revised: 7 September 2007 - Accepted: 12 September 2007 - Published: 14 September 2007

\begin{abstract}
An infinite number of unstable periodic orbits (UPOs) are embedded in a chaotic system which models some complex phenomenon. Several algorithms which extract UPOs numerically from continuous-time chaotic systems have been proposed. In this article the damped NewtonRaphson-Mees algorithm is reviewed, and some important techniques and remarks concerning the practical numerical computations are exemplified by employing the Lorenz system.
\end{abstract}

\section{Introduction}

Complex phenomena concerning geophysics, space physics and fluid dynamics are often described by chaotic dynamical systems with continuous time. An infinite number of unstable periodic orbits (UPOs) embedded in a chaotic system play important roles in characterizing and analyzing the system. In some continuous-time chaotic systems, several UPOs are numerically found. For example, Kazantsev $(1998,2001)$ detected UPOs of a barotropic ocean model, and discussed sensitivity of the attractor of the model to external influences by using them. Rempel and Chian (2005) and Chian, Kamide, Rempel and Santana (2006) discussed Alfven intermittency in space plasma dynamics through UPOs. Kawahara and Kida (2001), Kato and Yamada (2003), van Veen, Kawahara and Kida (2005) extracted UPOs in fluid dynamics models and showed that they characterize turbulence properties. However, even numerically, it is not easy to detect many UPOs from a continuous-time chaotic system, because they cannot be found by the forward time integration of the system. Then studies on numerical methods and techniques of identifying UPOs are important, and several numerical algorithms have been proposed so far. The most popular

Correspondence to: Y. Saiki

(saiki@kurims.kyoto-u.ac.jp) algorithm of finding UPOs is the Newton-Raphson method with a Poincaré section, which reduces a continuous dynamical system to a discrete system. Another method is to detect a UPO by stabilizing the periodic orbit in the sense of chaos control (Pyragas, 1992) ${ }^{1}$. Kazantsev (1998) develops a method which requires similar techniques to data assimilation, and there is also a variational method (Lan and Cvitanović, 2004). However, in this paper, we focus our attention to the Newton-Raphson-Mees method (Mees, 1981; Parker and Chua, 1989) with a damping coefficient, where we do not need to have trouble of choosing the appropriate Poincaré section.

When this algorithm is employed at the practical numerical computation for the purpose of detecting many UPOs, it is necessary to select the suitable damping coefficient. The coefficient is taken to distill the actual error of the initial guess from the targeted UPO in each iteration of the NewtonRaphson-Mees algorithm. There is the best coefficient for detecting each UPO effectively. If the employed damping coefficient is weaker than the best value, the UPO cannot be found. And if the employed damping coefficient is stronger, it takes much time to converge to the UPO. In this paper we discuss the damping coefficient in detail in relation to the stability exponent and the period, by taking the Lorenz system as an example.

A periodic orbit numerically detected by this algorithm is numerically valid in the sense that the trajectory of a point close to the periodic orbit remains close to the orbit for a whole period. It is also confirmed in this article that this algorithm can detect UPOs which are outside the attractor in addition to UPOs embedded in the attractor.

\footnotetext{
${ }^{1}$ For the Hénon map, there is an excellent method of detecting UPOs by identifying stable periodic orbits of the dual system (Biham and Wenzel, 1989), though its mathematical aspects are not well understood.
} 


\section{Algorithm for detecting UPOs}

\subsection{Algorithm}

The Newton-Raphson-Mees algorithm for detecting UPOs of the $n$-dimensional ordinary differential equations

$\frac{d \mathbf{x}}{d t}=F(\mathbf{x}), \mathbf{x} \in \mathbf{R}^{n}\left(\mathbf{C}^{n}\right)$

is described (Parker and Chua(1989)). $\left\{\phi_{t}(\mathbf{x})\right\}_{t \in \mathbf{R}}$ denotes the orbit passing through $\mathbf{x}\left(\mathbf{x} \in \mathbf{R}^{n}\left(\mathbf{C}^{n}\right)\right)$ at $t=0$. The periodic orbit of the system is determined by the zeros $(\mathbf{x}, t)=(\mathbf{X}, T)(T>0)$ of

$H(\mathbf{x}, t):=\phi_{t}(\mathbf{x})-\mathbf{x}$,

where $\mathbf{X}$ is one point on the periodic orbit and $T$ is the period. The normal Newton-Raphson algorithm does not work directly for solving this problem, because

$H(\mathbf{x}, t)=0$

has $n$ equations with $n+1$ unknowns: the $n$ components of $\mathbf{X}$ on the periodic orbit and the period $T$. The numerical algorithm is as follows. Linearizing $H(\mathbf{x}, t)$, we obtain

$$
\begin{aligned}
\Delta H(\mathbf{x}, t) & =D_{\mathbf{x}} H(\mathbf{x}, t) \Delta \mathbf{x}+D_{t} H(\mathbf{x}, t) \Delta t \\
& =\left\{\Phi_{t}(\mathbf{x})-I\right\} \Delta \mathbf{x}+F\left(\phi_{t}(\mathbf{x})\right) \Delta t,
\end{aligned}
$$

where $D_{\mathbf{x}} H$ and $D_{t} H$ indicate the variations of $H$ about $\mathbf{x}$ and $t$, respectively. Moreover, $\Phi_{t}(\mathbf{x})$ is $n \times n$ matrix and represents the variation of $\phi_{t}(\mathbf{x})$ about $\mathbf{x}$, and $I$ is the $n \times n$ unit matrix. $\Delta \mathbf{x}$ and $\Delta t$ are determined so as to satisfy $H(\mathbf{x}, t)+\Delta H(\mathbf{x}, t)=0$, namely

$\left\{\Phi_{t}(\mathbf{x})-I\right\} \Delta \mathbf{x}+F\left(\phi_{t}(\mathbf{x})\right) \Delta t=-H(\mathbf{x}, t)$.

Since this has $n$ constraints with $n+1$ unknowns, $\Delta \mathbf{x}$ and $\Delta t$ are not determined uniquely. Then one additional constraint is required in order to obtain a unique solution of the system. The constraint proposed by Mees (1981) is that the correction vector $\Delta \mathbf{x}$ is to be orthogonalized to the orbit, i.e.,

$$
<F(\mathbf{x}), \Delta \mathbf{x}>=0 .
$$

We can detect periodic orbits by iterating the procedure (6) and (7) several times. That is, under an appropriate initial guess $(\mathbf{x}, t)=\left(\mathbf{X}^{(i)}, T^{(i)}\right)$, we solve the equation about $\Delta \mathbf{X}^{(i)}$ and $\Delta T^{(i)}$,

$$
\begin{aligned}
& \left(\begin{array}{cc}
\Phi_{T^{(i)}}\left(\mathbf{X}^{(i)}\right)-I & F\left(\phi_{T^{(i)}}\left(\mathbf{X}^{(i)}\right)\right) \\
F\left(\mathbf{X}^{(i)}\right)^{t} & 0
\end{array}\right)\left(\begin{array}{c}
\Delta \mathbf{X}^{(i)} \\
\Delta T^{(i)}
\end{array}\right) \\
& =\left(\begin{array}{c}
\mathbf{X}^{(i)}-\phi_{T^{(i)}}\left(\mathbf{X}^{(i)}\right) \\
0
\end{array}\right),
\end{aligned}
$$

and modify the initial guess as

$$
\left(\mathbf{X}^{(i+1)}, T^{(i+1)}\right)=\left(\mathbf{X}^{(i)}, T^{(i)}\right)+2^{-m}\left(\Delta \mathbf{X}^{(i)}, \Delta T^{(i)}\right),
$$

where superscripts are added to indicate the iteration count denoted by $i(i \in \mathbf{N}), 2^{-m}$ is the damping coefficient, and $m(m \in \mathbf{N})$ the damping parameter.

\subsection{Convergence criterion}

We consider two sorts of errors in iterating the above algorithm:

$\operatorname{err}_{\text {prac }}^{(i)}:=\left|H\left(\mathbf{X}^{(i)}, T^{(i)}\right)\right|=\left|\phi_{T^{(i)}}\left(\mathbf{X}^{(i)}\right)-\mathbf{X}^{(i)}\right|$,

$\operatorname{err}_{\text {mod }}^{(i)}:=\left|\left(\Delta \mathbf{X}^{(i)}, \Delta T^{(i)}\right)\right|$.

The former is a practical error at the $i$-th iteration which is the distance between the initial point $\mathbf{X}^{(i)}$ and the point $\phi_{T^{(i)}}\left(\mathbf{X}^{(i)}\right)$. The latter is the absolute value of the modified vector $\left(\Delta \mathbf{X}^{(i)}, \Delta T^{(i)}\right)$. We consider an unstable periodic orbit to be numerically detected if both errors are sufficiently small at the $i$-th iteration. In this case, $X^{(i)}$ is considered a point on the UPO and $T^{(i)}$, its period. The errors are regarded as sufficiently small if

$\operatorname{err}_{\text {prac }}^{(i)} \ll 1, \quad \operatorname{err}_{\text {mod }}^{(i)} \ll 1$.

It is obvious from the criteria of the convergence of the algorithm that we cannot obtain UPOs which does not satisfy the condition $e^{\lambda T} \ll 1$, where $\lambda$ is the Floquet exponent and $T$ is the period. Note that the terms "converge" and "convergence" in this paper are used in the numerical sense discussed above.

\subsection{Initial guess}

Before iterating the algorithm, it is necessary to give the initial guess $\left(\mathbf{X}^{(0)}, T^{(0)}\right)$. For the convergence of the algorithm it is very important to give an appropriate initial guess. We randomly choose $\mathbf{X}^{(0)}$ on the numerically calculated chaotic orbits, and give $T^{(0)}$ randomly. This is for the purpose of detecting various types of UPOs.

\section{UPOs embedded in the chaotic attractor of the Lorenz system}

For examining practical aspects of numerical computations of the Newton-Raphson-Mees method, we employ the Lorenz system (Lorenz, 1963; Sparrow, 1982):

$\frac{d x}{d t}=\sigma(y-x), \frac{d y}{d t}=r x-y-x z, \frac{d z}{d t}=x y-b z$

$(\sigma=10, b=8 / 3, r=28)^{2}$ as an example of continuous-time chaotic systems. The system has a reflection symmetry with respect to $x \rightarrow-x, y \rightarrow-y, z \rightarrow z$ and fixed points $\left(x^{*}, y^{*}, z^{*}\right)=(0,0,0),( \pm \sqrt{b(r-1)}, \pm \sqrt{b(r-1)}, r-1)(r \geq 1)$. The classical Lorenz system includes an infinite number of UPOs, which are densely distributed in the chaotic attractor (Tucker, 1999, 2001). There are several studies on UPOs of the Lorenz system. For example, Eckhardt and Ott (1994) and Wiklund and Elgin (1996) studied the zeta function,

\footnotetext{
${ }^{2} r$ is set as 23 in Sect. 4 .
} 

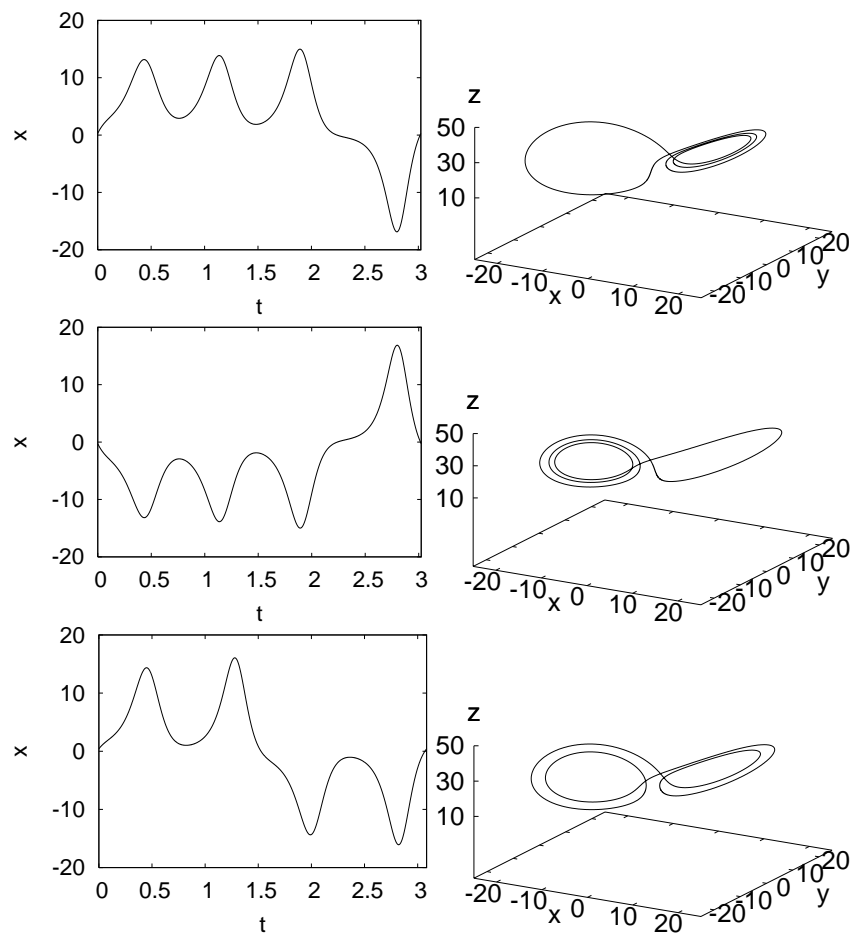

Fig. 1. Time series (left) of $x$ variable and projections (right) of detected UPOs with PERIOD4 ( $T=3.023583$ (top), 3.023583 (middle), 3.084276 (center)) (The top and the middle UPOs are identifiable based on the reflection symmetry).

the Hausdorff dimension and $f(\alpha)$ spectrum. Franceschini, Giberti and Zheng (1993) and Vithwanath (2003, 2004) detected UPOs in a systematic way and suggested that all the UPOs are labeled by a sequence of symbols, while Zoldi (1998) examined statistical properties of the Lorenz system at both classical and non-classical parameter values.

\subsection{Detected UPOs}

More than 1000 UPOs are extracted numerically from the chaotic attractor of the classical Lorenz system. Time series and projections of three UPOs are shown in Fig. 1. Here we briefly see some properties about UPOs of the system. It should be noted here that two types of period are used for each periodic orbit. One is the normal real number period (period $T$ ), and the other is the integer period (PERIOD $N_{T}$ ), which is the period of the Poincaré map whose Poincaré plane is $z=27$, and corresponds to the number of rotations around the two symmetric singular points.

\subsubsection{Number of UPOs}

We count the number of UPOs by the PERIOD and find the clear exponential growth of the number of UPOs (Fig. 2). Variations of periodic orbits indicate the topological com-

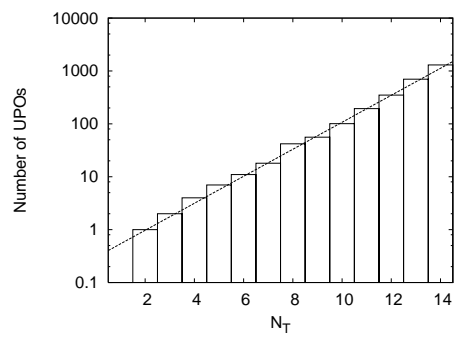

Fig. 2. Number of detected UPOs with PERIOD $N_{T}$ (Straight line is $1.8^{N_{T}}$ multiplied by the constant)
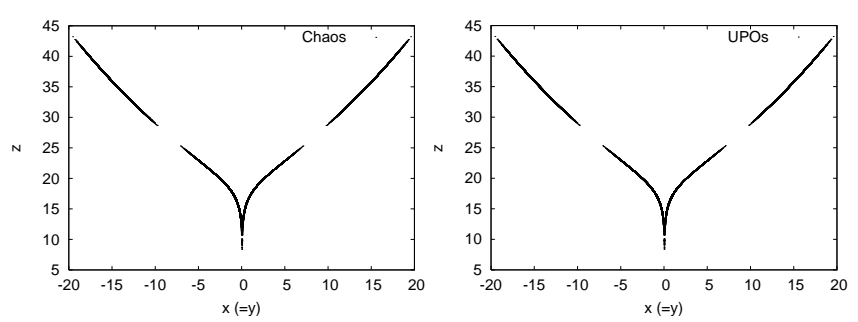

Fig. 3. Poincaré points on $x z$-plane at $x=y$ of chaotic orbits (left) and UPOs (right).

plexity of the system, and are closely related with the topological entropy (Bowen, 1970). If we find the growth rate of the number of UPOs by covering all or almost all UPOs with low periods, we can estimate the topological complexity of the system.

\subsubsection{Set of UPOs and chaotic attractor}

UPOs are densely embedded in a chaotic attractor of the classical Lorenz system (Tucker, 1999, 2002). In fact we can confirm the "complete" correspondence between Poincaré points of chaotic orbits and those of UPOs on $x z$-plane at $x=y$ in Fig. 3. This correspondence is common in chaotic systems and explains why typical dynamical properties along chaotic orbits can be captured by using dynamics along UPOs detected numerically (Kawahara and Kida, 2001; Kato and Yamada, 2003; Ishiyama and Saiki, 2005; Saiki and Ishiyama, $2007^{3}$ ).

\subsection{Practical numerical computations for detecting UPOs}

\subsubsection{Damping coefficient associated with Floquet expo- nent and period of UPOs}

The importance of damping coefficients for finding many UPOs by the convergence method like the Newton-Raphson

\footnotetext{
${ }^{3}$ Saiki, Y. and Ishiyama, K.: Unstable periodic orbits as the skeleton of a chaotic oscillator, Business cycles, in preparation, 2007.
} 

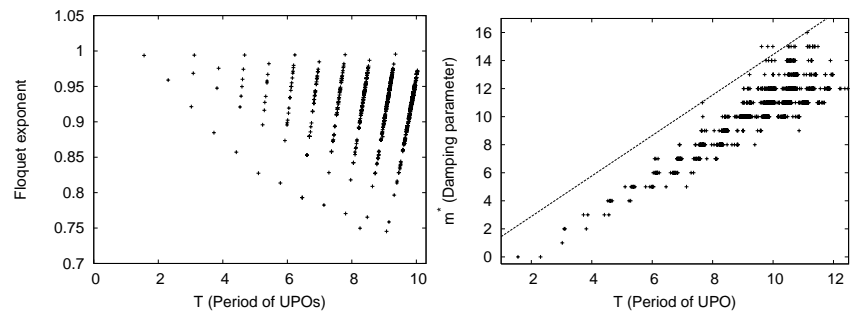

Fig. 4. Floquet exponent of each UPO with period $T$ (the Floquet exponents of UPOs with PERIOD $N$ are on the same "straight line") (left), Required damping parameter $m$ for detecting each UPO with period $T$ (straight line indicates $1.4427 \mathrm{~T}$. The damping parameter needed to detect a UPO tends to increase as the period increases) (right).

method has already been pointed out by Zoldi and Greenside (1998). In fact we can detect only a few UPOs, when we do not employ damping coefficients. But as far as we know, there are no discussions about how to decide the damping coefficient for the appropriate detection of UPOs.

We propose from a simple consideration of the algorithm that the damping parameter $m$ be selected according to the stability exponent $\lambda$ (Floquet exponent) and the period $T$ of a periodic orbit of the dynamical system. If the distance from the periodic orbit to the initial guess $\left(\mathbf{X}^{(i)}, T^{(i)}\right)$ at the $i$-th iteration in the Newton-Raphson-Mees algorithm is $\delta(\ll 1)$, this error grows to about $\delta e^{\lambda T^{(i)}}$ at time $T^{(i)}$ by the exponential instability of the system. The damping coefficient is adopted to distill the genuine error of the initial point from the nearest point on a UPO in each iteration. Then the appropriate value of the damping coefficient is thought of as $1 / e^{\lambda T^{(i)}}$ from the above physical consideration. Then the damping parameter $m$ in Eq. (10) should be chosen as

$m \approx \lambda T^{(i)} / \log 2 \approx 1.4427 \lambda T^{(i)}$.

It should be noted that this damping coefficient is essentially different from that often used in the Newton-Raphson method adopted to find the solution of a normal algebraic equation.

The approximate period of the periodic orbit to be detected is known in advance, but the stability exponent such as Floquet exponent (Chicone, 2006) is unknown. Hence it is necessary to try some damping coefficients according to the given initial guess for the detection of each targeted UPO. Figure 4 (left) shows the Floquet exponent of each UPO with period $T$ detected numerically. The exponent is determined through calculating the Floquet multiplier (maximal eigenvalue) of the UPO of the corresponding Poincare map. We can find that the upper bound of the Floquet exponent of UPO with PERIOD $N$ nearly equals to 1 , which is independent of $N$. Figure 4 (right) shows the required (lower limit of) damping parameter $m^{*}$ to detect each UPO with period $T$ by the damping Newton-Raphson-Mees method. $m^{*}$ for each UPO
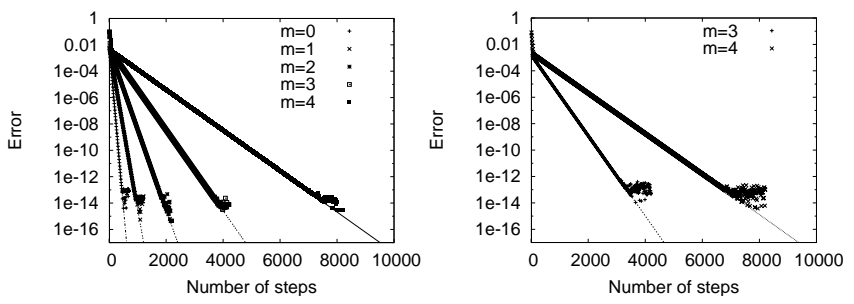

Fig. 5. Practical error at the $i$-th step $\left(\operatorname{err}_{\mathrm{prac}}^{(i)}\right)$ in the converging process to each UPO ( $T=1.55865$ (left), 4.41777 (right)) for various damping parameters $m(m=0,1,2,3,4)$ ( $m$ should be chosen to be more than 2 for identifying the latter UPO).

is identified by trying some computations for convergence of the damped Newton-Raphson-Mees method with the same initial guess at different values of $m \in \mathbf{Z}$. From the figure we can confirm that the required damping parameter $m^{*}$ for detecting a UPO with period $T$ tends to increase as $T$ increases. The result is consistent with the estimation (Eq. 15), which indicates the linear dependence of the required damping parameter on the period if the Floquet exponent $\lambda$ is fixed, and the slope is estimated to be about $1.4427 \lambda$. Note that rigorously speaking the required damping parameter $m\left(=m^{*}\right)$ for converging to each UPO depends on the initial condition nearby the orbit. Required damping parameter $m^{*}$ for detecting even the same UPO depends on the initial guess, but the difference is usually at most one from our experiences.

\subsubsection{Number of steps required for convergence to UPOs}

We consider the number of steps required to converge to UPOs. The number of steps in the Newton-Raphson-Mees algorithm before identifying a UPO is much bigger than our expectation, even if we choose the appropriate damping parameter $m\left(=m^{*}\right)(\in \mathbf{N})$, which is the lowest damping parameter for convergence in the algorithm. Figure 5 shows the practical error at $i$-th iteration $\operatorname{err}_{\text {prac }}^{(i)}$ of the NewtonRaphson-Mees algorithm with the damping parameter $m$ for two UPOs ( $T=1.55865,4.41777)$. The latter UPO is an example of UPOs which we cannot extract when we do not adopt the idea of damping coefficients. When we choose $m$ as a damping parameter, the number of steps required to converge to each UPO is about $2^{m-m^{*}}$ times as many as that when we choose $m^{*}$ which is the lowest damping parameter for convergence. The targeted UPO cannot be identified, if we choose $m\left(\leq m^{*}-1\right)$ as a damping parameter.

\subsection{Validity of numerically detected UPOs}

Here we discuss the numerical validity of a UPO identified by the Newton-Raphson-Mees method. Figure 6 shows the error of the period of a detected UPO from the "genuine" pe$\operatorname{riod}\left(\operatorname{err}_{T}=\left|T(\mathrm{dt})-T_{0}\right|\right)$, where $\mathrm{dt}$ is the time step of a discretization in the integration by the 4th order Runge-Kutta 


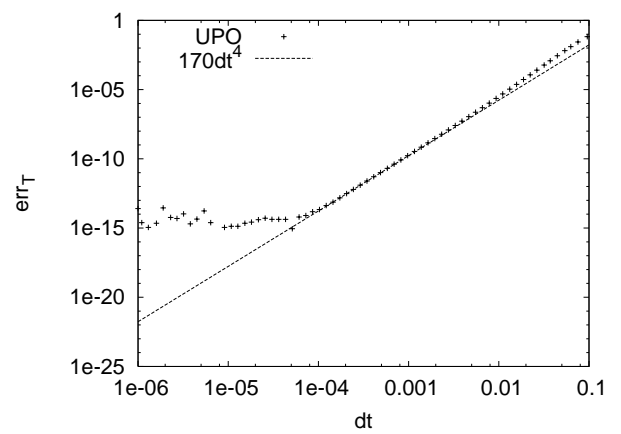

Fig. 6. The error of the time-step-dependent period of a numerically detected UPO $\left(\operatorname{err}_{T}=\left|T(\mathrm{dt})-T_{0}\right|\right)$, where $\mathrm{dt}$ is the time step of the integration by the Runge-Kutta method, $T_{0}$ is the "genuine" period which is estimated from the computation with the quadrupleprecision arithmetic with a sufficiently small time step.

method, and the "genuine" period is estimated from the numerically robust period, as long as the time steps are sufficiently small. In this case, the numerical accuracy of the period of the UPO is $O\left(10^{-15}\right)$, as our computation is in double precision.

The error $\operatorname{err}_{T}$ is proportional to $(\mathrm{dt})^{4}$ for the range $(0.0001<\mathrm{dt}<0.01)$, which is consistent with the fact that we use the 4th order Runge-Kutta method. This supports the validity of the numerical computation of the UPO. Note that the property is not satisfied at $\mathrm{dt}<0.0001$ in the case of doubleprecision arithmetic because of the round off error.

\section{UPOs outside of the attractor of Lorenz system}

Some studies on chaotic saddles (Nusse and Yorke, 1989) of dynamical systems have been done so far. Recently chaotic saddles of the complex system described by a PDE, which generates spatio-temporal chaotic behavior, also have been found by Rempel and Chian (2007). However, chaotic saddles are usually identified not by UPOs but by indirect methods, the sprinkler (Kantz and Grassberger, 1985; Hsu et al., 1988 ) or the PIM triple method (Nusse and Yorke, 1989). Here we detect chaotic saddles of the Lorenz system $(r=23)$ by extracting UPOs directly.

It should be noted that the attracting sets of the Lorenz system with $r=23$ are composed of two fixed points. Before reaching the attractor, the orbits from most initial conditions behave chaotically. And an infinite number of UPOs are embedded in the nonattracting chaotic set (chaotic saddles), which is thought to be responsible for chaotic transient dynamics. We show the projections of chaotic transients before reaching to the attractor and the set of numerically detected UPOs. In fact we can see the close similarity between chaotic transient behavior and the set of UPOs from the Poincare points of $x z$-plane at $x=y$ in Fig. 7. We should remark that
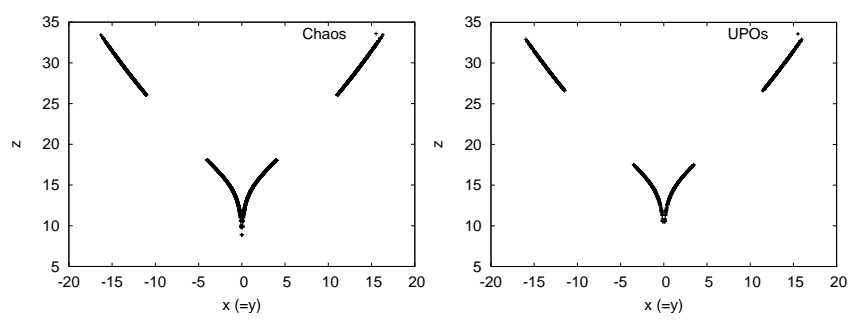

Fig. 7. Poincaré points on $x z$-plane at $x=y$ of chaotic transient behaviors (left) and UPOs (right) at $r=23$.

initial guesses should be chosen nearby the numerically calculated chaotic behavior in detecting UPOs.

\section{Conclusions}

The Newton-Raphson-Mees method with a damping coefficient, which can detect a lot of unstable periodic orbits (UPOs) of a chaotic system of continuous-time, is reviewed with the detailed procedures and remarks on numerical computation by taking the Lorenz system as an example. We consider how to choose the appropriate damping coefficient and find that it can be determined by the Floquet exponent and the period of each UPO for extracting various types of many UPOs effectively. The numerical validity of periodic orbits detected by the Newton-Raphson-Mees method is confirmed in relation to the error which depends on the time step in discretization. It is also exemplified that many UPOs outside the attractor, which form chaotic saddles, can be detected in addition to UPOs embedded in the attractor by employing the method. This suggests that the method is valid not only for analyzing the chaotic attractor but for the nonattracting chaotic set (chaotic transient) through detecting various sorts of UPOs.

One of the greatest advantage of the Newton-RaphsonMees method is that we do not have trouble with choosing the appropriate Poincaré plane which crosses orbits of the system. Another advantage is that a UPO detected by this method is the numerically robust orbit. A disadvantage is that the sorts of UPOs which can be detected by this method are limited by the instability and the period of UPOs and the numerical accuracy, which is, however, related to the former advantage. This is a very difficult but an inevitable problem to be solved for building better algorithm. Moreover, for moving on to the next stage, we have to establish criteria for distinguishing or identifying numerical detected close UPOs automatically.

Acknowledgements. The author is very thankful to the anonymous referees for their significant comments and suggestions. This work is partly supported by the 21 st Century COE program at the University of Tokyo and Keio University and Grant-in-Aid for JSPS Fellows. 
Edited by: A. C. L. Chian

Reviewed by: two anonymous referees

\section{References}

Biham, O. and Wenzel, W.: Characterization of unstable periodic orbits in chaotic attractors and repellers, Phys. Rev. Lett., 63, 819-822, 1989.

Bowen, R.: Topological entropy and AxiomA, Global Analysis (Berkeley, CA, 1968) Proc. Sympos. Pure Math., 14, Amererican Mathathematical Society, Providence, RI, 23-41, 1970.

Chian, A. C. L., Kamide, Y., Rempel, E. L., and Santana, W. M.: On the chaotic nature of solar-terrestrial environment: interplanetary Alfven intermittency, J. Geophys. Res., 111(10), A07S03, doi:10.1029/2005JA011396, 2006.

Chicone, C.: Ordinary Differential Equations with Applications, Springer-Verlag, 1999.

Eckhardt, B. and Ott, E.: Periodic orbit analysis of the Lorenz attractor, Zeitschrift fur Physik B Condensed Matter, 93(2), 258266, 1994.

Franceschini, V., Giberti, C., and Zheng, Z.: Characterization of the Lorenz attractor by unstable periodic orbits, Nonlinearity, 6(2), 251-258, 1993.

Gratrix, S. and Elgin, J. N.: Pointwise Dimensions of the Lorenz Attractor, Phys. Rev. Lett., 92, 014101:1-4, 2004.

Hsu, G. H., Ott, E., and Grebogi, C.: Strange saddles and the dimension of their invariant manifolds, Phys. Lett. A, 127, 199-204, 1988.

Ishiyama, K. and Saiki, Y.: Unstable periodic orbits and chaotic economic growth, Chaos, Solitons and Fractals, 26, 33-42, 2005.

Kantz, H. and Grassberger, P.: Repellers, semi-attractors, and longlived chaotic transients, Physica D, 17, 75-86, 1985.

Kato, S. and Yamada, M.: Unstable periodic solutions embedded in a shell model turbulence, Physical Review E, 68(2), 25302:1-4, 2003.

Kawahara, G. and Kida, S.: Periodic motion embedded in plane Couette turbulence: regeneration cycle and burst, J. Fluid Mechanics, 449, 291-300, 2001.

Kazantsev, E.: Unstable periodic orbits and attractor of the barotropic ocean model, Nonlin. Processes Geophys., 5, 193208, 1998,

http://www.nonlin-processes-geophys.net/5/193/1998/.
Kazantsev, E.: Sensitivity of the attractor of the barotropic ocean model to external influences: approach by unstable periodic orbits, Nonlin. Processes Geophys., 8, 301-311, 2001, http://www.nonlin-processes-geophys.net/8/301/2001/.

Lan, Y. and Cvitanović, P.: Variational method for finding periodic orbits in a general flow, Phys. Rev. E, 69(1), 016217, 2004.

Lorenz, E. N.: Deterministic nonperiodic flow, J. Atmos. Sci., 20, 130-141, 1963.

Mees, A. I.: Dynamics of Feedback Systems, John Wiley and Sons, New York, N. Y., 1981.

Nusse, H. E. and Yorke, J. A.: A procedure for finding numerical trajectories on chaotic saddles, Physica D, 36, 137-156, 1989.

Parker, T. S. and Chua, L. O.: Practical Numerical Algorithms for Chaotic Systems, Springer-Verlag, 1989.

Pyragas, A.: Continuous control of chaos by selfcontrolling feedback, Phys. Lett. A, 170, 421-428, 1992.

Rempel, E. L. and Chian, A. C.-L.: Space plasma dynamics: Alfven intermittent Chaos, Adv. Space Res., 35, 951-960, 2005.

Rempel, E. L. and Chian, A. C.-L.: Origin of Transient and Intermittent Dynamics in Spatiotemporal Chaotic Systems, Phys. Rev. Lett., 98, 14101:1-4, 2007.

Sparrow, C.: The Lorenz equations: Bifurcations, chaos, and strange attractors, Springer-Verlag, 1982.

Tucker, W.: The Lorenz attractor exists, Comptes Rendus de l'Académie des Sciences, Série I. Mathmatique, 328:12, 1197$1202,1999$.

Tucker, W.: A rigorous ODE solver and Smale's 14th problem, Foundations of Computational Mathematics, 2, 53-117, 2002.

van Veen, L., Kida, S., and Kawahara, G.: Periodic motion representing isotropic turbulence, Fluid Dynam. Res., 38(1), 19-46, 2006.

Viswanath, D.: Symbolic dynamics and periodic orbits of the Lorenz attractor, Nonlinearity, 16, 1035-1056, 2003.

Viswanath, D.: The fractal property of the Lorenz attractor, Physica D, 190, 115-128, 2004.

Wiklund, K. O. and Elgin, J. N.: Multifractality of the Lorenz system, Phys. Rev. E, 54(2), 1111-1119, 1996.

Zoldi, S.: Unstable Periodic Orbit Analysis of Histograms of Chaotic Time Series, Phys. Rev. Lett., 81, 3375-3378, 1998.

Zoldi, S. M. and Greenside, H. S.: Spatially localized unstable periodic orbits of a high-dimensional chaotic system, Phys. Rev. E, 57(3), 2511-2514, 1998. 